\title{
Surface Plasmon-Polaritons and Transverse Spin Angular Momentum at the Boundary of Hyperbolic Metamaterial with Arbitrary Orientation of the Optical Axis
}

\author{
Vladimir Belyi ${ }^{*}$, Svetlana Kurilkina ${ }^{1}$, Nikolai Kazak ${ }^{1}$, \\ and Vladimir Agabekov ${ }^{2}$
}

\author{
${ }^{1}$ B.I. Stepanov Institute of Physics of National Academy of Sciences of Belarus, Minsk, Belarus \\ ${ }^{2}$ Institute of Chemistry of New Materials of National Academy of Sciences of Belarus, Minsk, Belarus \\ *corresponding author, E-mail: v . belyi@ifanbel . bas - net . by
}

\begin{abstract}
The possibility is established and the conditions are found for localization of plasmon-polaritons (PPs) near the boundaries of hyperbolic metamaterials (HMs) of both I and II types with arbitrary orientation of the optical axis. It is grounded that such surface PP has the transverse spin momentum which depends on the wavelength of the exciting wave, the orientation of the optical axis of the hyperbolic metamaterial, and dielectric properties of bordered media.
\end{abstract}

\section{Introduction}

In the general case the angular momentum, carried by the propagating monochromatic light beam, represents a superposition of two longitudinal components, the directions of which coincide with the wave vector [1]: spin angular momentum (SAM) and orbital angular momentum (OAM) related to circular polarization of the beam and its phase profile in the section orthogonal to the propagation direction, respectively. Vortex (Laguerre-Gaussian, Bessel-Gaussian) circularly polarized beams with the amplitude of electric vector $E(\rho, \varphi)=E_{o}(\rho) \exp (\operatorname{im} \varphi) \quad(\rho, \varphi$ are the cylinder coordinates, $m$ is the azimuthal phase index, or topological charge) are the examples of the fields possessing both SAM and OAM. For these vortex beams each photon carries OAM that equals to $m \hbar$ and SAM which has a value $\pm \hbar$ depending on the sign of circular polarization. For linear polarized propagating beam the longitudinal spin angular momentum is absent [1-3]. During the interaction of vortex beams with microobjects the transfer of spin and orbital momenta to the particles takes place that permits to control them.

Recently it has been shown that in contrast to the case of propagating waves the spin momentum of evanescent waves (including ones with linear polarization) appears to be nonzero and perpendicular to the wave vector [4,5]. Thus, the transverse SAM arises, the origin of which is caused by the rotation of the electric vector of the evanescent wave in the propagation plane. The features of transverse SAM are investigated for evanescent waves at the boundary of isotropic media. But optical anisotropy can essentially influence the conditions of existence of surface waves and transverse spin related to them.

Last decade hyperbolic metamaterials (HMM) have attracted significant scientific interests due to their extraordinary properties and great prospects for the subwavelength imaging [6,7], the control of spontaneous emission [8], the thermal radiative heat transfer [9]. In Ref. [10] the possibility is shown of the presence of transverse spin momentum for plasmon-polariton fields excited at the boundary of the II type hyperbolic metamaterial with optical axis perpendicular to this boundary. Recently it has been shown that the hyperbolic metamaterials with tilted optical axes exhibit asymmetry properties for waves propagating upward and downward with respect to slab interfaces $[11,12]$ that is potentially prospective for creation of a new types of optical devices, for example, an absorber [11], meta-waveguides and sensors [12], a wave expander and a source shifter [13]. Asymmetric hyperbolic media can be fabricated from different materials such as aligned carbon nanotubes, graphene, multilayers metal-dielectric structures, aligned noble nanotubes implemented into a dielectric matrix [1113]. In this paper we investigate the influence of HMM optical axis orientation on the conditions of existence of surface plasmon-polaritons with transverse spin momentum. As an example, we consider HMM created from the multilayered metal-dielectric structure.

\section{Surface plasmon-polariton at the boundary of isotropic medium and hyperbolic metamaterial with arbitrary oriented optical axis}

2.1. Features of plasmon-polaritons at the boundary of isotropic medium and hyperbolic metamaterial with arbitrary oriented optical axis

Let us consider a metamaterial formed from layeredperiodic metal-dielectric medium. In effective medium theory when the thickness of each layer is sufficiently small, i.e. $\left|k_{d} d_{d}\right|<<1,\left|k_{m} d_{m}\right|<<1$ where $k_{d}, k_{m}$ are the wave numbers of dielectric and metallic layers, 
respectively, this multilayered structure can be considered as anisotropic effective medium with the permittivity tensor $\varepsilon$ represented in the form:

$$
\varepsilon=\operatorname{diag}\left\{\varepsilon_{\perp}, \varepsilon_{\perp}, \varepsilon_{\|}\right\}=\varepsilon_{\perp}+\delta \mathbf{c} \otimes \mathbf{c},
$$

where $\delta=\varepsilon_{\|}-\varepsilon_{\perp}, \varepsilon_{\|}$and $\varepsilon_{\perp}$ are the longitudinal (along the $Z^{\prime}$ axis) and transverse (in the plane orthogonal to $Z^{\prime}$ ) main permittivities, $\mathbf{c}$ is the unit vector along the HMM optical axis, and the symbol $\otimes$ denotes the dyadic product of the vectors $\left(\mathbf{c} \otimes \mathbf{c}=c_{i} c_{k}\right)$. The main permittivities of this structure are determined by the following relations:

$$
\varepsilon_{\perp}=(1-f) \varepsilon_{d}+f \varepsilon_{m}, \varepsilon_{\|}^{-1}=\left[(1-f) / \varepsilon_{d}+f / \varepsilon_{m}\right] .
$$

Here $\varepsilon_{d}$ and $\varepsilon_{m}$ permittivity of dielectric and metallic layer, respectively, $f$ is the filling factor (the volume part of metal in the unit cell). The permittivity of metal $\varepsilon_{m}$ is described by the modified Drude formula:

$$
\begin{aligned}
& \varepsilon_{m}(\omega)=\varepsilon_{\infty}-\omega_{p}^{2} /\left(\omega^{2}+i \omega \Gamma\right)= \\
& =\varepsilon_{\infty}-\omega_{p}^{2} /\left(\omega^{2}+\Gamma^{2}\right)+i \omega_{p}^{2} \Gamma /\left[\omega\left(\omega^{2}+\Gamma^{2}\right)\right] .
\end{aligned}
$$

Here $\omega_{p}$ is the volume plasma frequency, $\varepsilon_{\infty}$ is the sum of the interband contributions, $\Gamma=V_{F} / l$ is the damping constant, $V_{F}$ is the Fermi velocity, $l$ is the electron mean free path in bulk metal. For silver we have $\varepsilon_{\infty}=5$, $\omega_{p}=14 \cdot 10^{15} s^{-1}, \quad \Gamma=32 \cdot 10^{12} s^{-1}, \quad V_{F}=1.4 \cdot 10^{6} \mathrm{~ms}^{-1}[14]$. For simplicity of our consideration we support that in Eq. (2) $\operatorname{Im}\left(\varepsilon_{t}\right) \approx 0, \quad \operatorname{Im}\left(\varepsilon_{l}\right) \approx 0 . \quad$ As calculation shows [15] this assumption is correct for spectral regions where parameters $\varepsilon_{\perp}$ and $\varepsilon_{\|}^{-1}$ significantly differ from zero.

Let the HMM borders with the isotropic medium having the permittivity $\varepsilon_{1}$. Meanwhile, the angle between the optical axis of the metamaterial and the normal to the HMM boundary ( $\mathrm{Z}$ axis) is $\theta$ (Fig.1). For definition we'll suggest that the optical axis is disposed between the $\mathrm{Z}$ and $\mathrm{X}$ axes of chosen coordinate system.

Now we consider $p$-polarized surface waves propagating along the $X$ axis in the plane separated the isotropic dielectric with the permittivity $\varepsilon_{1}$ and hyperbolic metamaterial. We'll use the coordinate system $X Y Z$ (see Fig.1) in which the effective permittivity tensor $\varepsilon^{\prime}$, characterizing the hyperbolic metamaterial, is represented in the form:

$$
\varepsilon^{\prime}=U \varepsilon \widetilde{U}
$$

Here $U$ is the transformation matrix which permits to transfer from the coordinate system related to the HMM main dielectric axes to the system of coordinate $X Y Z$ :

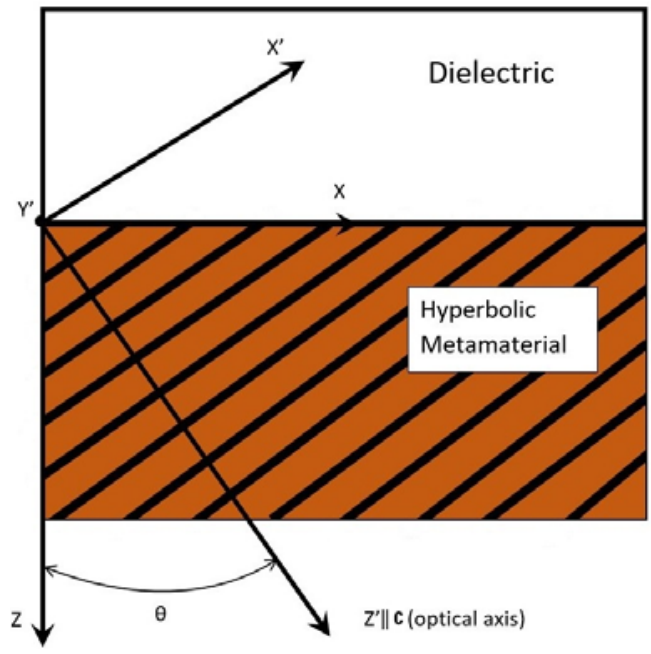

Figure 1: Schematic view of the hyperbolic metamaterial slab bordered with the dielectric. The $Z^{\prime}$ axis is parallel to the c vector.

$$
U=\left(\begin{array}{ccc}
\cos \theta & 0 & \sin \theta \\
0 & 1 & 0 \\
-\sin \theta & 0 & \cos \theta
\end{array}\right)
$$

the symbol "tilde" denotes the transposition. As follows from Eqs.(4) and (1)

$$
\begin{aligned}
& \varepsilon^{\prime}=U \varepsilon \widetilde{U}=\varepsilon_{x x} \mathbf{e}_{x} \otimes \mathbf{e}_{x}+\varepsilon_{\perp} \mathbf{e}_{y} \otimes \mathbf{e}_{y}+\varepsilon_{z z} \mathbf{e}_{z} \otimes \mathbf{e}_{z}+ \\
& +\varepsilon_{x z}\left(\mathbf{e}_{x} \otimes \mathbf{e}_{z}+\mathbf{e}_{z} \otimes \mathbf{e}_{x}\right) .
\end{aligned}
$$

Here

$$
\begin{aligned}
& \varepsilon_{x x}=\varepsilon_{\perp} \cos ^{2} \theta+\varepsilon_{\|} \sin ^{2} \theta, \\
& \varepsilon_{z z}=\varepsilon_{\perp} \sin ^{2} \theta+\varepsilon_{\|} \cos ^{2} \theta, \\
& \varepsilon_{x z}=\left(\varepsilon_{\|}-\varepsilon_{\perp}\right) \sin \theta \cos \theta,
\end{aligned}
$$

$\mathbf{e}_{x}, \mathbf{e}_{y}$, and $\mathbf{e}_{z}$ are the unit vectors of used coordinate system.

Now we represent the vectors of the field inside the dielectric $(d)$ and metamaterial $(m)$ in the form

$$
\mathbf{F}=\left\{\begin{array}{rc}
\mathbf{F}^{d} \exp \left(\kappa_{d} z+i q x-i \omega t\right), & z<0, \\
\mathbf{F}^{m} \exp \left(-\kappa_{m} z+i q x-i \omega t\right), & z>0
\end{array},\right.
$$

where $\mathbf{F}$ denotes electric $\mathbf{E}=\left(E_{x}, 0, E_{z}\right)$ or magnetic $\mathbf{H}=\left(0, H_{y}, 0\right)$ vector, $\kappa_{d, m}>0$ and $q$ are the decay constants and longitudinal wave number of the surface wave (plasmon-polariton), respectively, $\quad \kappa_{d}^{2}=q^{2}-k_{0}^{2} \varepsilon_{1}$, $k_{0}=\omega / c, \omega$ is the cyclic frequency of electromagnetic wave, $c$ is the light velocity in vacuum. Taking into account Eq. (8) from the Maxwell equations one can obtain the expressions for electric $\mathbf{E}^{d}, \mathbf{E}^{m}$ and magnetic

$\mathbf{H}^{d}, \mathbf{H}^{m}$ vectors inside the dielectric and metamaterial: 


$$
\begin{aligned}
& \mathbf{E}^{d}=-\frac{A_{0}}{\varepsilon_{1}}\left(i \frac{\kappa_{d}}{k_{0}} \mathbf{e}_{x}+\frac{q}{k_{0}} \mathbf{e}_{z}\right) e^{i q x+\kappa_{d} z}, \\
& \mathbf{H}^{d}=A_{0} \mathbf{e}_{y} e^{i q x+\kappa_{d} z} . \\
& \mathbf{E}^{m}=\frac{A_{0}}{s k_{0}}\left[\left(i \kappa_{m} \varepsilon_{z z}+q \varepsilon_{x z}\right) \mathbf{e}_{x}-\right. \\
& \left.-\left(q \varepsilon_{x x}+i \kappa_{m} \varepsilon_{x z}\right) \mathbf{e}_{z}\right] e^{i q x-\kappa_{m} z}, \\
& \mathbf{H}^{m}=A_{0} \mathbf{e}_{y} e^{i q x-\kappa_{m} z} .
\end{aligned}
$$

Here $s=\varepsilon_{x x} \varepsilon_{z z}-\varepsilon_{x z}^{2}, A_{0}$ is the amplitude, and the phase factor exp $-i \omega t$ is omitted. Substituting Eq. (10) into the Maxwell equation $r o t \mathbf{E}=-(1 / c) \partial \mathbf{H} / \partial t$ we find:

$$
\kappa_{m}^{2}=a q^{2}-b k_{0}^{2}+2 i q d \kappa_{m} .
$$

Here

$$
a=\varepsilon_{x x} / \varepsilon_{z z}, b=s / \varepsilon_{z z}, d=\varepsilon_{x z} / \varepsilon_{z z} .
$$

If $d$ parameter is small the decay constant $\kappa_{m}$ is represented in the form:

$$
\kappa_{m}=\kappa_{m o}+i \kappa_{m 1} .
$$

It should be noted that $\left|\kappa_{m o}\right|>>\left|\kappa_{m 1}\right|$. Then, squaring Eq. (12) and comparing the result with Eq. (11), we obtain:

$$
\kappa_{m}=\kappa_{m o}+i d q=\sqrt{a q^{2}-b k_{0}^{2}}+i d q .
$$

It follows from Eq. (13) that the decay constant $\kappa_{m}$ is complex. Thereby, the surface wave has nonzero propagation constant along the normal to the metamaterial boundary. It means that its phase velocity is directed under the $\gamma$ angle towards the HMM boundary:

$$
\tan \gamma=-d=-\frac{\left(\varepsilon_{\|}-\varepsilon_{\perp}\right) \sin \theta \cos \theta}{\varepsilon_{\perp} \sin ^{2} \theta+\varepsilon_{\|} \cos ^{2} \theta} .
$$

As follows from Eq. (14), two different cases of orientation of the phase velocity of the located wave can be realized (Fig.2). As seen from Fig.2, for these two cases the planes of equal phase and equal amplitudes are not collinear. For the I type $\operatorname{HMM}\left(\varepsilon_{\perp}>0, \varepsilon_{\|}<0\right)$ we have

$$
d=\frac{\sin 2 \theta}{2\left(\frac{\left|\varepsilon_{\|}\right|}{\left|\varepsilon_{\|}\right|+\varepsilon_{\perp}}-\sin ^{2} \theta\right)} .
$$

For the II type HMM $\left(\varepsilon_{\perp}<0, \varepsilon_{\|}>0\right)$ parameter $d$ is represented in the form

$$
d=\frac{\sin 2 \theta}{2\left(\frac{\varepsilon_{\|}}{\varepsilon_{\|}+\left|\varepsilon_{\perp}\right|}-\sin ^{2} \theta\right)} .
$$

As follows from Eqs. (15), (16), if $0<\theta<\pi / 2$ the parameter $d$ is positive (and the phase velocity is directed towards the dielectric) for the following range of angles:

$\sin \theta<\sqrt{\frac{\left|\varepsilon_{\|}\right|}{\left|\varepsilon_{\|}\right|+\varepsilon_{\perp}}}$ (for the I type HMM),
$\sin \theta<\sqrt{\frac{\varepsilon_{\|}}{\varepsilon_{\|}+\left|\varepsilon_{\perp}\right|}}$ (for the II type HMM).

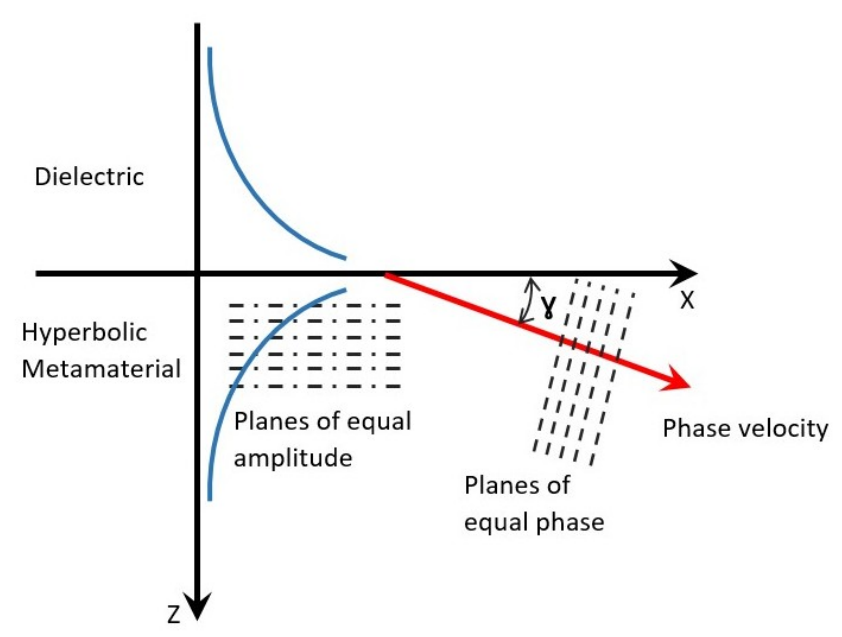

(a)

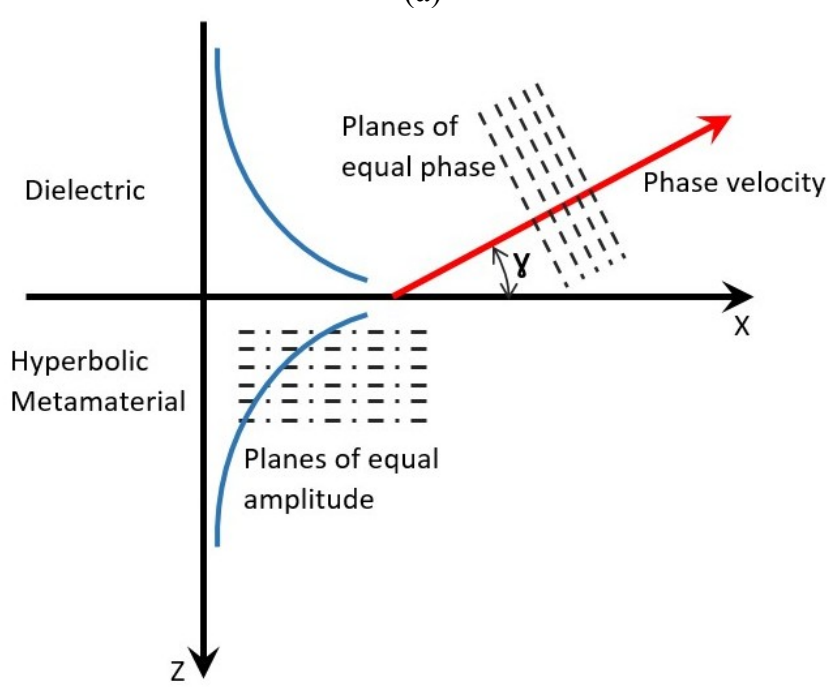

(b)

Figure 2: Orientation of the phase velocity of located wave at the boundary of hyperbolic metamaterial for the case of negative (a) and positive (b) values of the parameter $d$.

From the boundary conditions for electric $\mathbf{E}^{d}, \mathbf{E}^{m}$ and magnetic $\mathbf{H}^{d}, \mathbf{H}^{m}$ vectors (see Eqs. (9), (10)) it follows the dispersion equation for plasmon-polariton: 


$$
\frac{1}{s}\left(i \kappa_{m o} \varepsilon_{z z}+q \varepsilon_{x z}\right)=-\frac{1}{\varepsilon_{1}}\left(i \kappa_{d}\right) .
$$

If $\theta \cong 0$ or $\theta \cong \pi / 2$ form Eq. (18) it follows the simple expression for the longitudinal wave number of the plasmon-polariton:

$$
q=k_{0} \sqrt{\frac{\varepsilon_{1} \varepsilon_{z z}\left(\varepsilon_{x x}-\varepsilon_{1}\right)}{\varepsilon_{x x} \varepsilon_{z z}-\varepsilon_{1}^{2}}}=k_{0} \sqrt{\varepsilon_{e f f}}
$$

\subsection{The condition of the plasmon-polariton localization at the boundary of hyperbolic metamaterial}

Now we'll analyze the possibility of localization of the electromagnetic wave on the interface of an isotropic medium and a hyperbolic metamaterial. Due to the exponentially decaying of the amplitude of this field when moving off the boundary, the wave number $q$ has to be larger than modules of wave vectors of the waves propagating inside the dielectric and metamaterial. Thereby,

$$
\varepsilon_{e f f}>0, \varepsilon_{e f f}>\varepsilon_{1}, \kappa_{m}>0 .
$$

From inequalities (20) the conditions of localization of plasmon-polariton at the boundary of hyperbolic metamaterial follows: $\varepsilon_{z z}>\varepsilon_{1}, \varepsilon_{x x}<0$. For the hyperbolic metamaterial of the I type these conditions are fulfilled at

$$
\begin{aligned}
& \sin ^{2} \theta>Y_{1}=\sin ^{2} \Theta_{1}=\frac{\varepsilon_{\perp}}{\left|\varepsilon_{\|}\right|+\varepsilon_{\perp}}, \\
& \sin ^{2} \theta>Y_{2}=\sin ^{2} \Theta_{2}=\frac{\left|\varepsilon_{\|}\right|+\varepsilon_{1}}{\left|\varepsilon_{\|}\right|+\varepsilon_{\perp}} .
\end{aligned}
$$

From Eqs.(21), (22) it follows the condition of existence of localized plasmon-polaritons at the boundary of the hyperbolic metamaterial of the I type and isotropic dielectric:

$$
\begin{aligned}
& |\theta|>\theta_{c 1}=\max \left\{\left|\Theta_{1}\right|,\left|\Theta_{2}\right|\right\}, \\
& \Theta_{1,2}=\arcsin \sqrt{Y_{1,2}} .
\end{aligned}
$$

Similarly, for the hyperbolic metamaterial of the II type the excited plasmon-polariton is localized if the following conditions are fulfilled:

$$
\begin{aligned}
& \sin ^{2} \theta<Y_{3}=\sin ^{2} \Theta_{3}=\frac{\left|\varepsilon_{\perp}\right|}{\varepsilon_{\|}+\left|\varepsilon_{\perp}\right|}, \\
& \sin ^{2} \theta<Y_{4}=\sin ^{2} \Theta_{4}=\frac{\varepsilon_{\|}-\varepsilon_{1}}{\varepsilon_{\|}+\left|\varepsilon_{\perp}\right|} .
\end{aligned}
$$

From Eqs. (24), and (25) one can obtain the general condition for existence of localized plasmon-polaritons at the boundary of the metamaterial of the II type and isotropic dielectric:

$$
\begin{aligned}
& |\theta|<\theta_{c 2}=\min \left\{\left|\Theta_{3}\right|,\left|\Theta_{4}\right|\right\}, \\
& \Theta_{3,4}=\arcsin \sqrt{Y_{3,4}} .
\end{aligned}
$$

Obtained Eqs. (23), (26) determine the conditions of localization of surface plasmon-polaritons at the interface of the isotropic medium and hyperbolic metamaterial.

\subsection{Localized plasmon-polaritons at the boundary of the multilayered metal-dielectric $\mathrm{Ti}_{3} \mathrm{O}_{5} / \mathrm{Ag}$ nanostructure}

As an example we consider the hyperbolic metamaterial created on the basis of the layered-periodic $\mathrm{Ti}_{3} \mathrm{O}_{5} / \mathrm{Ag}$ structure the unit cell of which is formed by the nanolayers of silver (Ag) and isotropic dielectric $\mathrm{Ti}_{3} \mathrm{O}_{5}$.

In Figs.3, 4 the spectral dependences of effective permittivities $\varepsilon_{\perp}$ and $\varepsilon_{\|}$are represented for hyperbolic metamaterial on the basis of the metal-dielectric layered $\mathrm{Ti}_{3} \mathrm{O}_{5} / \mathrm{Ag}$ structure. For calculation here and below we suggested that the thickness of the metal layer was $20 \mathrm{~nm}$, and the filling factor was $f=0.3$. As is seen from Figs.3,4, in the spectral region of $320 \mathrm{~nm}<\lambda<390 \mathrm{~nm}$ this structure displays the properties of the I type HMM, and for the wavelength $\lambda>600 \mathrm{~nm}$ it can be considered as the II type HMM.

Now we suggest that all layers are deposited on the substrate (for example, fused quartz with dielectric permittivity $\varepsilon_{1}=2.1$ ), and the periodicity axis (the optical axis) of the structure is oriented at certain angle $\theta$ to the normal to its border. As is illustrated in Figs.5,6, for this case the condition (23) is fulfilled for large values of $\theta$ $(\theta \rightarrow \pi / 2)$. Meanwhile, if the wavelength $\lambda$ increases (up to $390 \mathrm{~nm})$ the value of the angle $\theta_{\mathrm{cl}}=\max \left\{\Theta_{1}, \Theta_{2}\right\}$ increases too (Fig.5).

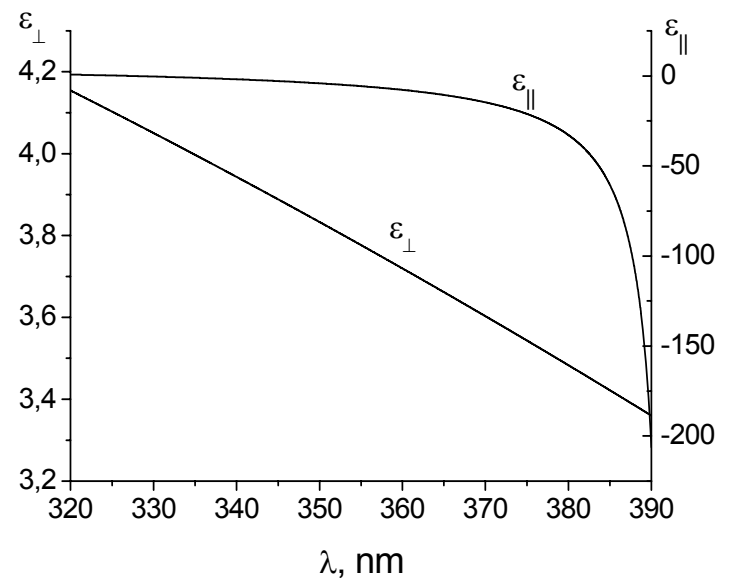

Figure 3: The spectral dependences of main dielectric permittivities of the I type HMM on the basis of multilayered $\mathrm{Ti}_{3} \mathrm{O}_{5} / \mathrm{Ag}$ nanostructure. 
For hyperbolic metamaterial of the II type created on the basis of the metal-dielectric layered $\mathrm{Ti}_{3} \mathrm{O}_{5} / \mathrm{Ag}$ structure the condition (26) is fulfilled at small values of the $\theta$ angle, i.e. when the optical axis is close to the normal to the HMM boundary (see Figs.7,8). Meanwhile, the parameter $\theta_{\mathrm{c} 2}=$ $\min \left\{\Theta_{3}, \Theta_{4}\right\}=\Theta_{3}$ increases with the growth of the wavelength $\lambda$ (Fig.7).

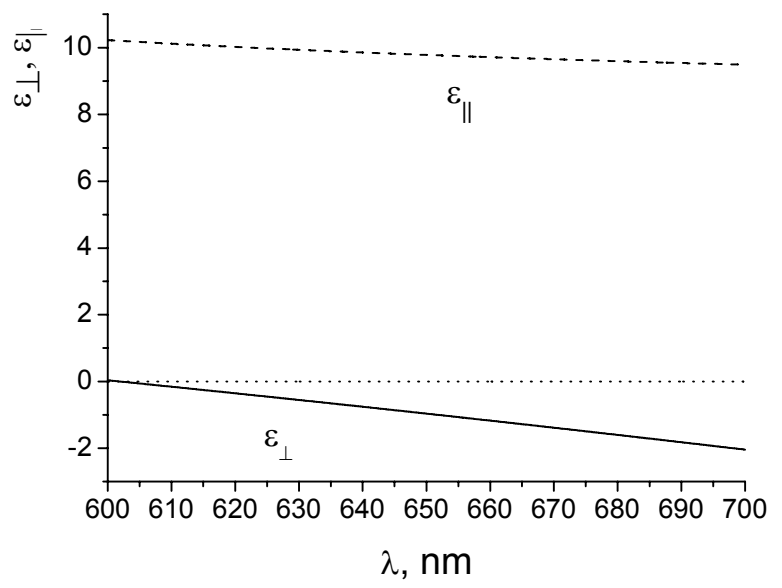

Figure 4: The spectral dependences of main dielectric permittivities of the II type HMM on the basis of multilayered $\mathrm{Ti}_{3} \mathrm{O}_{5} / \mathrm{Ag}$ nanostructure.

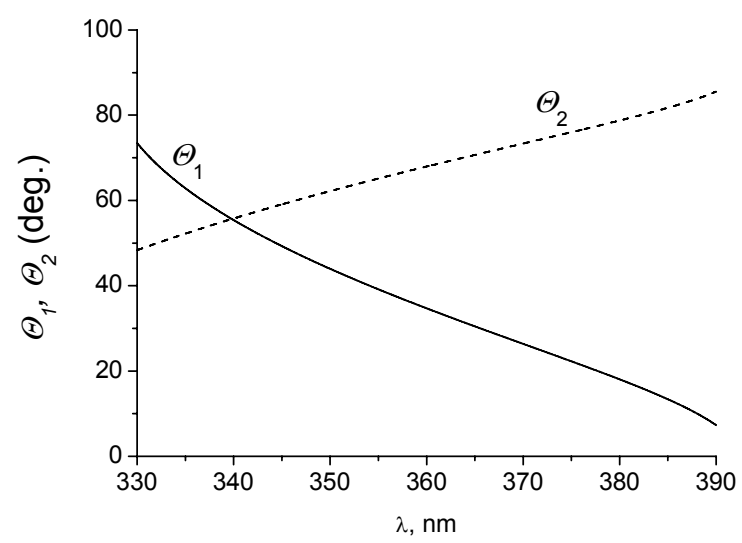

Figure 5: The spectral dependences of the angles $\Theta_{1,2}=\arcsin \left(\mathrm{Y}_{1,2}\right)^{1 / 2}$ for the I type HMM on the basis of multilayered $\mathrm{Ti}_{3} \mathrm{O}_{5} / \mathrm{Ag}$ nanostructure.

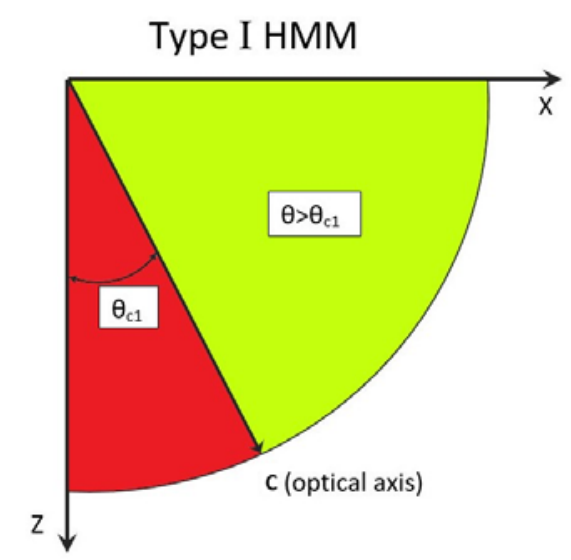

Figure 6: Schematic view of the range of angles $\left(\theta>\theta_{c 1}\right)$ for which localized plasmon-polaritons exist at the boundary of the I type HMM on the basis of $\mathrm{Ti}_{3} \mathrm{O}_{5} / \mathrm{Ag}$ nanostructure.

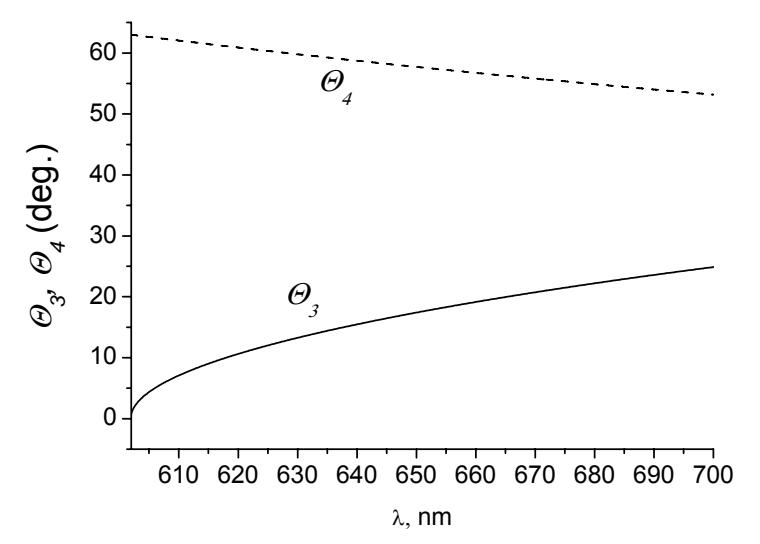

Figure 7: The spectral dependences of the angles $\Theta_{3,4}=\arcsin \left(\mathrm{Y}_{3,4}\right)^{1 / 2}$ for the II type HMM on the basis of multilayered $\mathrm{Ti}_{3} \mathrm{O}_{5} / \mathrm{Ag}$ nanostructure.

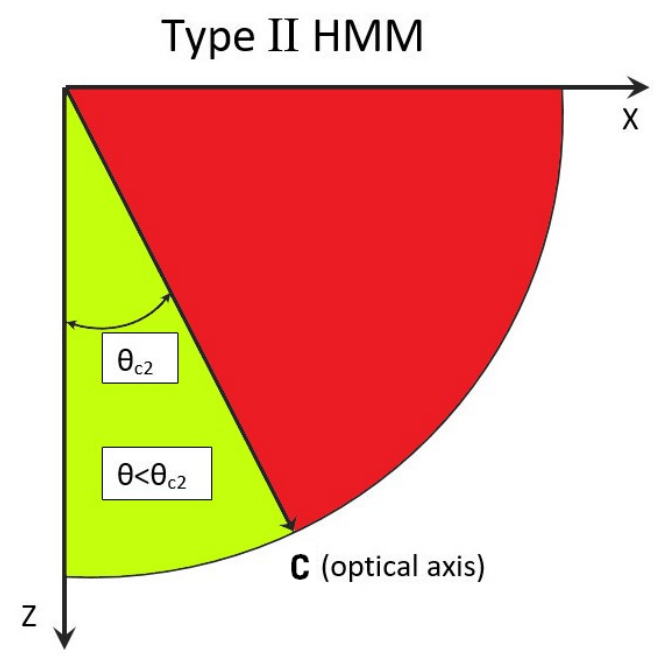

Figure 8: Schematic view of the range of angles $\left(\theta<\theta_{c 2}\right)$ for which localized plasmon-polaritons exit at the boundary of the II type HMM on the basis of multilayered $\mathrm{Ti}_{3} \mathrm{O}_{5} / \mathrm{Ag}$ nanostructure. 


\section{Transverse spin angular momentum of surface plasmon-polariton}

It is known that p-polarized wave possesses the spin angular momentum, expected value $\mathbf{S}$ of which (in $\hbar$ units per photon) is determined by relation $[2,16,17]$ :

$$
\mathbf{S}=\frac{\operatorname{Im}\left(\mathbf{E}^{*} \times \mathbf{E}\right)}{\mathbf{E}^{*} \cdot \mathbf{E}}
$$

As follows from Eq. (9), the electric vector of plasmonpolariton inside the dielectric $\mathbf{E}^{d}=E_{x}^{d} \mathbf{e}_{\mathbf{x}}+E_{z}^{d} \mathbf{e}_{\mathbf{z}}$ has two components (real $E_{z}^{d}=-q A_{0} /\left(k_{0} \varepsilon_{1}\right)$ and imaginary $E_{x}^{d}=-\kappa_{d} A_{0}\left(k_{0} \varepsilon_{1}\right)$ parts) lying in the propagation plane $(X Z)$. Owing to this the phase difference $-\pi / 2$ between the components $E_{x}^{d}$ and $E_{z}^{d}$ appears. This causes the rotation of the electric vector in the propagation plane $(X Z)$ and emergence of the transverse component $S_{y}$ of spin angular momentum. As follows from Eq. (27), in general case the expression for $S_{y}$ has the form:

$$
S_{y}=-2 \frac{\operatorname{Re} E_{x}^{d} \operatorname{Im} E_{z}^{d}-\operatorname{Im} E_{x}^{d} \operatorname{Re} E_{z}^{d}}{\left|E_{x}^{d}\right|^{2}+\left|E_{z}^{d}\right|^{2}} .
$$

For the considered case when $\operatorname{Im} E_{z}^{d}=\operatorname{Re} E_{x}^{d}=0$ from Eq. (28) we obtain:

$$
S_{y}=\frac{2 \kappa_{d} q}{q^{2}+\kappa_{d}^{2}} .
$$

Eq. (29) is convenient to use for calculating the amplitude of the transverse component $S y$ of the spin as a function of the wavelength of the surface plasmon-polariton at the interface between a hyperbolic metamaterial and a dielectric (in particular, water (or another liquid) containing microparticles). In this case, the parameter $S y$ determines the torque momentum acting in the direction of the $Y$-axis on particles near the surface of the metamaterial.

In Fig. 9 the dependences of the expected value of transverse spin (in units of $\hbar$ per photon) on the wavelength and the angle $\theta$ for plasmon-polariton localized at the boundary of layered-periodic medium $\mathrm{Ti}_{3} \mathrm{O}_{5} / \mathrm{Ag}$ and fused quartz are represented.

It is seen that the expected value $S_{y}$ of transverse spin changes with variation of the wavelength. This indicates the possibility of controlling the transverse spin momentum by changing the wavelength of the surface plasmon-polariton.

However, as is seen in Fig.9, the dependences $S_{y}(\lambda)$ are deferent for the I and II types of HMM. For the I type HMM (Fig.9a) the expected value of transverse spin is small $\left(S_{y}<0.27\right.$ ), whereas for the II type HMM the value of $\mathrm{S}_{\mathrm{y}}$ approaches unity (Fig.9b).

Moreover, in Figs.10,11 it is shown that if the wavelength increases from the short-wavelength region (where the structure possesses the properties of the I type HMM) up to the wavelength for which $\varepsilon_{\|}^{-1} \rightarrow 0$, the expected $S_{y}$ value decreases (Fig.10). If the wavelength decreases from the long-wavelength region (where the structure possesses the properties of the II type HMM) up to $\lambda$ for which $\varepsilon_{\perp} \rightarrow 0$, the expected value $S_{y}$ of transverse spin momentum increases (Fig.11).

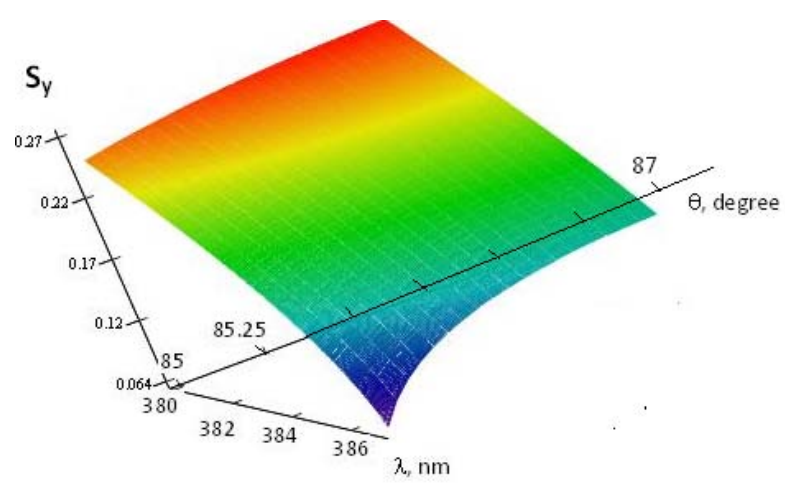

(a)

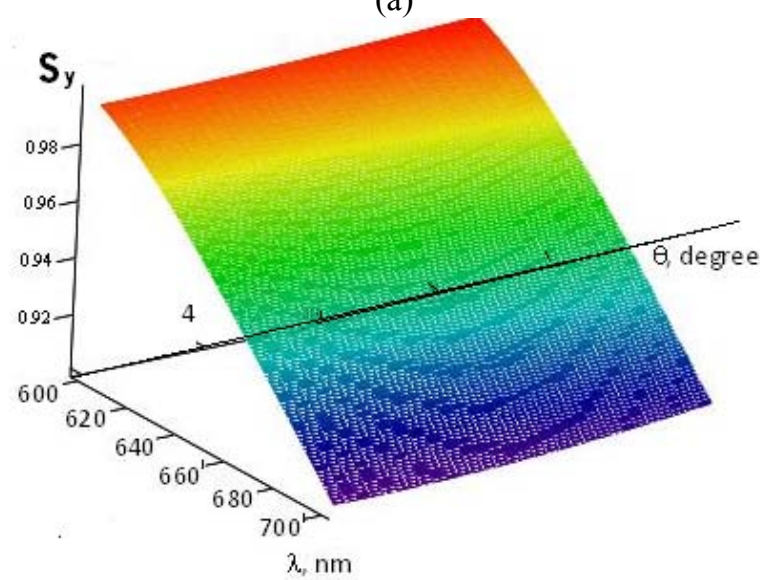

(b)

Figure 9: Expected value of transverse spin (in $\hbar$ units per photon) for the plasmon-polariton localized at the interface of a dielectric (fused quartz) and hyperbolic metamaterial of the I type (a) and II type (b). Hyperbolic metamaterial is formed from the multilayered $\mathrm{Ti}_{3} \mathrm{O}_{5} / \mathrm{Ag}$ nanostructure.

The expected value of transverse spin appears to depend on the orientation of the optical axis and permittivity of the medium bordered with the metamaterial. But these dependences are different for the I and II types of HMMs. In the first case (I type HMM) the dependence $S_{y}(\lambda)$ changes weakly with increasing the angle $\theta$ (Fig.10a). In the second case (II type HMM) with increasing the angle $\theta$ the expected value $S_{y}$ of transverse spin also increases (Fig.11a). If the permittivity $\varepsilon_{1}$ increases we also observe different changes in $S_{y}(\lambda)$ dependences. For the I type HMM the $S_{y}$ value decreases (Fig.10b), but for the II type HMM two spectral regions exist: for the first of them $(\lambda>640 \mathrm{~nm})$ the value of $S_{y}$ increases, and for the second one $(\lambda<640 \mathrm{~nm}) S_{y}$ decreases (Fig.11b). 


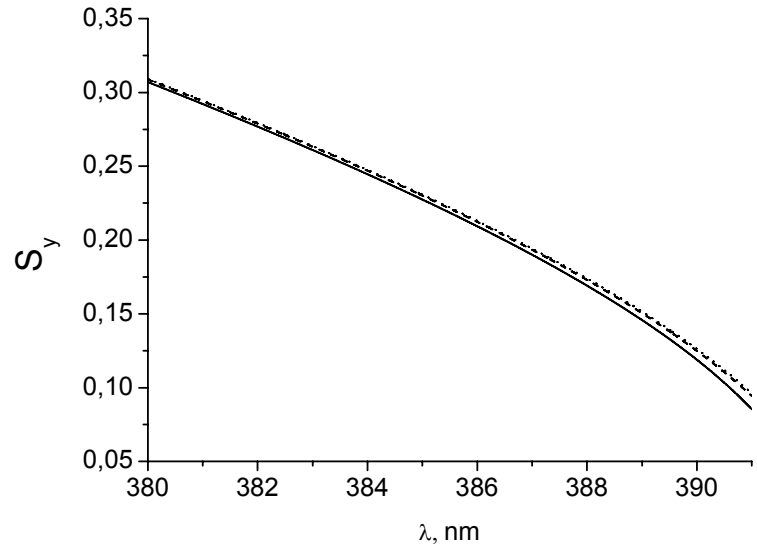

(a)

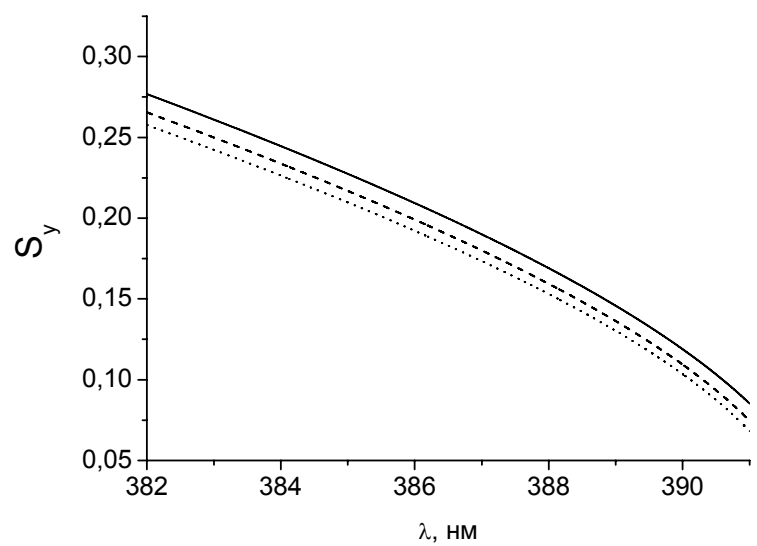

(b)

Figure 10: Spectral dependence of the expected value of transverse spin (in $\hbar$ units per photon) for the plasmonpolariton formed at interface of the metal-dielectric multilayered $\mathrm{Ti}_{3} \mathrm{O}_{5} / \mathrm{Ag}$ structure and a dielectric: a) water ( $\left.\varepsilon_{1}=1.769\right)$; b) water (solid line), glyserine ( $\left.\varepsilon_{1}=2.170\right)$ (dashed line), optical glass BAF 10 $\left(\varepsilon_{1}=2.753\right)$ (dotted line). The optical axis is oriented at the angle: a) $\theta=87.8^{\circ}$ (solid line), $89^{\circ}$ (dashed line), $90^{\circ}$ (dotted line); b) $87.8^{\circ}$.

\section{Conclusions}

Thus, in this paper we investigated the features of plasmonpolaritons excited at the interface of a hyperbolic metamaterial and a dielectric for the case when the optical axis is arbitrary oriented under the normal to the boundary. Expressions are obtained for the complex electric and magnetic vectors as well as for the decay constants of the fields on both sides of the interface. It is shown that the wave vector of plasmon-polariton has the component oriented perpendicular to the boundary. It is established that for hyperbolic metamaterials of different types (I and II) changing the orientation of the optical axis one can realize the conditions when the phase velocity of plasmon-polariton is directed from the boundary inside a metamaterial or a dielectric.

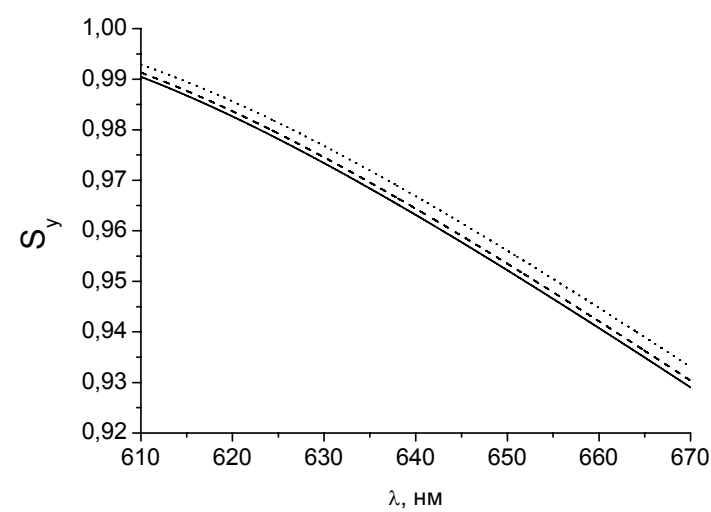

(a)

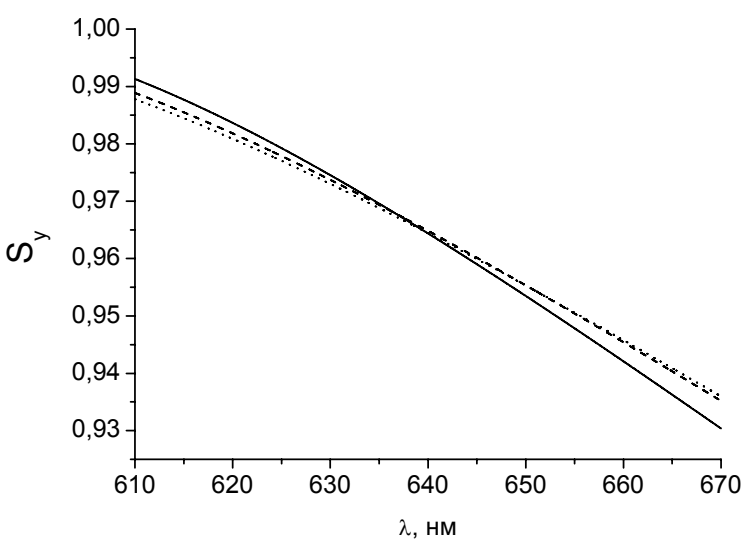

(b)

Figure 11: Spectral dependence of the expected value of transverse spin (in $\hbar$ units per photon) for plasmonpolariton formed at the boundary of the metal-dielectric multilayered $\mathrm{Ti}_{3} \mathrm{O}_{5} / \mathrm{Ag}$ structure and a dielectric: a) water ( $\left.\varepsilon_{1}=1.769\right)$; b) water (solid line), glyserine ( $\varepsilon_{1}=2.170$ ) (dashed lline), optical glass BAF 10 $\left(\varepsilon_{1}=2.753\right.$ ) (dotted line). Optical axis is oriented under the angle: a) $\theta=1^{\circ}$ (solid line), $3^{\circ}$ (dashed line), $5^{\circ}$ (dotted line); b) $3^{\circ}$.

The possibility is shown and the conditions are determined for localization of plasmon-polariton at the boundary of hyperbolic metamaterial of both the I and II types.

It is grounded that surface plasmon-polariton, localized near the interface of isotropic dielectric and hyperbolic metamaterial, possesses the transverse spin angular momentum, the expected value of which depends on the 
wavelength, the orientation of the optical axis of HMM, and permittivity of bordered medium.

For illustration of the obtained results the numerical modeling is carried out of the plasmon-polariton and transverse SAM properties for the case of hyperbolic metamaterial formed on the basis of the multilayered $\mathrm{Ti}_{3} \mathrm{O}_{5} / \mathrm{Ag}$ nanostructure.

\section{References}

[1] J. D. Jackson, Classical Electrodynamics, Wiley, Berkeley, 1999.

[2] M.V. Berry. Optical currents, J. Opt. A: Pure Appl. Opt. 11: 094001, 2009.

[3] A. Bekshaev, K.Y. Bliokh, M. Soskin, Internal flows and energy circulation in light beams, J. Opt. 13: 053001, 2011.

[4] K.Y. Bliokh, F. Nori, Transverse spin of a surface polariton, Phys. Rev. A 85: 061801, 2012.

[5] K.Y. Bliokh, A.Y. Bekshaev, F. Nori, Dual electromagnetism: helicity, spin, momentum and angular momentum, New J. Phys. 15: 033026, 2013.

[6] E. E. Narimanov, V. Shalaev. Beyond diffraction, Nature 447: 266-267, 2007.

[7] Z. Jacob, L. V. Alekseyev, E. E. Narimanov. Optical hyperlens: far-field imaging beyond the diffraction limit, Opt. Express 14: 8247-8256, 2006.

[8] M. A. Noginov et al. Controlling spontaneous emission with metamaterials, Optics Letters 35: 18631865, 2010.

[9] C. Simovski, S. Maslovski, I. Nefedov, S. Tretyakov, Optimization of radiative heat transfer in hyperbolic metamaterials for thermophotovoltaic applications, Opt. Express 21: 14988-15013, 2013.

[10] S.N. Kurilkina, V.N. Belyi, N.S. Kazak, Surface plasmon-polaritons and transverse spin angular momentum at the boundary of hyperbolic metamaterials, J Appl Specrosc 83: 965-969, 2017.

[11]I. Nefedov, C. Valagiannopoulos, S. Hashemi, E. Nefedov. Total absorption in asymmetric hyperbolic media, Scientific Reports 3: 2662, 2013.

[12]A. D. Boardman1, P. Egan, M. McCall. Optic axisdriven new horizons for hyperbolic metamaterials, EPJ Appl. Metamat. 2: 11, 2015.

[13] Z. Liang, X. Jiang, F. Miao, S. Guenneau, J. Li. Transformation media with variable optical axes, New Journal of Physics 14: 103042, 2012.

[14]W. Cai, V. Shalaev, Optical Metamaterials. Fundamentals and Applications, Springer, New York, 2010.

[15] S. N. Kurilkina, M. A. Binhussain, V. N. Belyi, N. S. Kazak, Features of hyperbolic metamaterials with extremal optical characteristics, J. Opt. 18: 085102, 2016.

[16] M.V. Berry, M.R. Dennis. Polarization singularities in isotropic random vector waves, Proc. R. Soc. London A.
Mathematical, Physical and Engineering Sciences 757: 141-155, 2001.

[17]A. Canaguier-Durand, C. Genet. Transverse spinning of a sphere in a plasmonic field, Phys. Rev. A 89: 033841, 2014. 